\title{
A Fog Networks for Measuring the Physical Parameter of Greenhouse Plant
}

\author{
Suryono Suryono ${ }^{1,2, *}$, Sunarno Sunarno ${ }^{3}$, Ragil Saputra ${ }^{4}$ \\ ${ }^{1}$ Magister of Information System, School of Postgraduate Study, Diponegoro University, Semarang - Indonesia \\ ${ }^{2}$ Department of Physics, Faculty of Science and Mathematics, Diponegoro University, Semarang - Indonesia \\ ${ }^{3}$ Department of Biology, Faculty of Science and Mathematics, Diponegoro University, Semarang - Indonesia \\ ${ }^{4}$ Department of Informatics, Faculty of Science and Mathematics, Diponegoro University, Semarang - Indonesia
}

\begin{abstract}
Greenhouse is a very effective method of matching and has been able to contribute to food independence in various countries. Plants that are in the greenhouse must be maintained with chemical-physical parameters in order to grow optimally. Monitoring of plants in greenhouses must always be done. Some monitoring reports have been made online so that they can provide solutions as quickly as possible if there is a disturbance on the plants. Unfortunately, online monitoring is still dependent on internet networks that require network infrastructure needs that have many limitations. As a result, many agricultural lands are not covered by the internet network to monitor the greenhouse. The use of a large number of sensor nodes also affects the decline in available broadband internet performance so as to reduce monitoring performance. In this research proposed a fog network that connects the sensor node with the local fog server via a WIFI network. Sensor node has been built with a system on chips WIFI-Microcontroller ESP8266 to perform data acquisition and temperature sensor data transmission, relative humidity and light intensity using the WIFI network to the fog server. In this study testing the accuracy of sensor parameters used and network performance by comparing with the use of cloud networks. From the tests performed, the results of Mean Absolute Percent Error (MAPE) were obtained for each parameter, temperature $=1.3 \%$, humidity: $1.9 \%$ and light intensity: $0.6 \%$. The use of the fog network has proven to not contribute significantly to the error value of measurement data sent to the server. The use of WIFI on the fog network requires less network broadband needs when compared to cloud networks. This difference is very significant, which is an average of 253 BPS if using a fog network and 1276 BPS if using a cloud network. From the experiments conducted, the use of networks for proven to have a high data transmission speed with value $471 \mathrm{~ms}$ when compared to the internet network with value $1349 \mathrm{~ms}$. Variations in the number of sensor nodes up to 5 nodes do not significantly affect that speed.
\end{abstract}

Keywords: monitoring ; online ; broadband ; sensor node ; transmission speed.

\section{Introduction}

Food security is an important issue for all nations, especially poor and developing countries. Food security is a very basic and essential problem because it is related to survival, health, nutrition and nutrition for long-term life in all countries [1]. The problem that arises to improve food security is the amount of land that has no productivity and far land from settlements due to industrialization [2]. Food security is being pursued by every country in the world in various ways. To achieve food security, collaboration is needed between science and technology products and social humanities. Therefore the development of science and technology in the agricultural industry continues to increase. Efforts to change from traditional agricultural systems to modern agricultural systems to improve agricultural yields are carried out by all countries in the world [3].
Measurement and control of chemical and physical parameters in a wide area requires the support of information technology, especially remote measurement technology which is more commonly called telemetry technology. The use of telemetry technology strongly supports the instrument system model for measurement and control to overcome terrain conditions that are widely distributed and represent samples. Therefore, the use of wireless technology is the only choice that is the best in the case [4].

Greenhouse is one of the effective farming techniques to adjust the climate to the needs of plants [5]. The technology applied to greenhouses can overcome environmental conditions that cause plant growth to be hampered [6]. Chemical and physical properties of the environment around plants greatly affect plant growth. Plant growth in the agricultural industry is strongly influenced by environmental factors [7]. Soil chemical and physical factors contribute greatly to the productivity

*Corresponding author: suryono@fisika.undip.ac.id 
of agricultural land. Environmental conditions and soil play a role as regulators of water flow and nutrient cycles for plants [3]. Conditioning of chemical and physical parameters is needed to optimize production results so that an effective and efficient measurement and control device is needed [8].

Measurement and control of chemical and physical parameters in greenhouses is very necessary so that the development of plants can grow optimally [9]. With the wireless sensor system model, this problem can be overcome because it simply represents the sensor in a field to automatically transmit data to the observation station. The constraints faced by wireless sensor systems are the limitations of networks, especially the internet for wireless computing and control.

In this study, a fog networking network model is proposed to overcome obstacles, especially delays, limitations of development, and further application. Fog networking provides a sensor network infrastructure that has high performance, data storage and network services [10]. Computing networking is proposed as one of the promising technologies for scalable network infrastructure development around users [11]. The fog network allows users to move the computing node closer to the object. This is because the fog network is close to the data source so that it can reduce the latency reduction [12].

\section{Related Work}

The use of wireless sensor systems has been growing rapidly because it is able to overcome spatial problems where sensors can be distributed in various locations and carried out data acquisition simultaneously and quickly to a station control. The use of wireless sensor system networks can unite sensor networks in a decentralized manner. The use of these networks requires efficient hardware and software to collect data in large capacity and software algorithms that can anticipate the system overload communication [13].

Fog networking is a local network that connects between sensors and cloud. In general, the fog network consists of sensor nodes located in the field, a fog server that collects data from the sensor. Data communication between sensors and fog servers can be done using a WIFI gateway that has high effectiveness and is independent [14]. The general fog networking architecture is shown in Figure 1.

Some microcontroller have been built using a WIFI network so that it is more effective and cheaper. The internet network is a global network that has limited access and speed of data transfer. With the support of the fog network, the problem can be overcome [15]. Fog Networking system has been developed to handle field conditions that have a fast data handling system. One example of this case is handling vehicle authentication with a solid road with an RFID sensor. This system has proven effective in addressing transportation security problems [16].
The fog network system that has the number of $\mathrm{N}$ and Ni nodes is the number of the node, the Time slot is notated $\mathrm{T}$, the network connection $\mathrm{s}$, the data latency is formulated:

$$
\mathrm{D}=\sum_{i=1}^{|N|} \sum_{j=1}^{|T|}\left[l_{i j}^{d} x\left(s_{i j}^{t}-s_{i j}\right)+l_{i j_{i j}}^{c} x S_{i j}\right]
$$

The total energy consumed by the network can be calculated using the formula:

$$
\mathrm{E}=\sum_{i=1}^{|N|} \sum_{j=1}^{|T|}\left(b_{i} x e_{i}^{d}+s_{i j} x e_{i}^{c}\right)
$$

The $e_{d}^{i}$ field shows the amount of energy in each node N. Fog computing can avoid busyness due to large amounts of data transfer because the computational node is in the area of the object that is close to the knot. The FOG network system is very useful for collecting large amounts of real-time streaming data and controlling conditions [17].

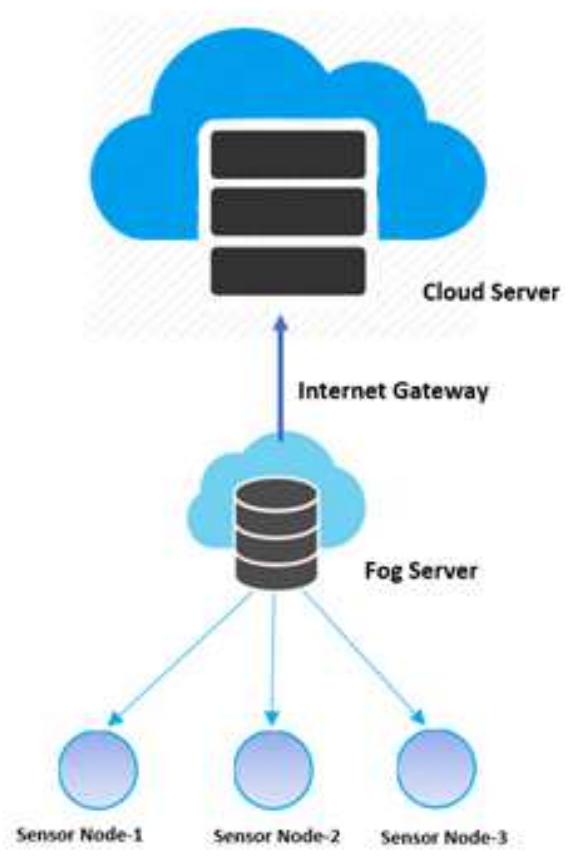

Fig. 1. General fog networking architecture.

\section{System Realization}

The monitoring system of the physical parameters of plants in a greenhouse using the fog network consists of a sensor node and a fog server that is connected to the cloud via the internet network. Sensor nodes are part of the fog system that functions to acquire data sensor and transmit data via WIFI radio. In this study, the system was built using ESP8255 microcontroller which has integrated 
the sensor data acquisition system and data communication system using WIFI. The sensors used to test this network are: temperature sensors, humidity sensors, and light intensity sensors. The fog network system built in this research is shown in Figure 2.

The sensor used is a sensor model whose reading value has been calibrated with from the manufacturer. In this study using a temperature sensor type SHT-11 which in one chip has a temperature parameter $\left({ }^{\circ} \mathrm{C}\right)$ and humidity (\%). Temperature sensor type SHT-11 requires TWI Two Wire Interface (TWI) protocol, which is a serial communication protocol that involves two cables called SDA (serial data) and SCL (serial clock). Other specifications owned by TWI are more flexible because they have master and slave modes, the address on the slave reaches 7-bit, and the data transmission speed reaches $400 \mathrm{KHz}$. The ESP8266 microcontroller is programmed to read the SHT11 sensor with the TWI protocol. Light sensors that have been used in this study are $\mathrm{BH}-1750$ type. The sensor has also been calibrated from the manufacturer and issued the reading of light intensity (Lux).

This communication network in fog networking uses 2 gateways, namely the WIFI network for data communication in the fog area and the internet network for data communication in the cloud area. To save and simplify the network, MIFI is used which includes an internet modem and wifi router. Internet mode functions to connect the fog server to the web server on the internet while the router function is to connect the sensor node to the fog server. The ESP8266 microcontroller is a System on Chips (SoC) which has been integrated between the microcontroller for data acquisition and WIFI for data communication. On this network, the ESP8266 microcontroller can be connected directly to the fog server via the wifi router on MIFI. The use of this system makes the device cheaper and more efficient in the power supply field.

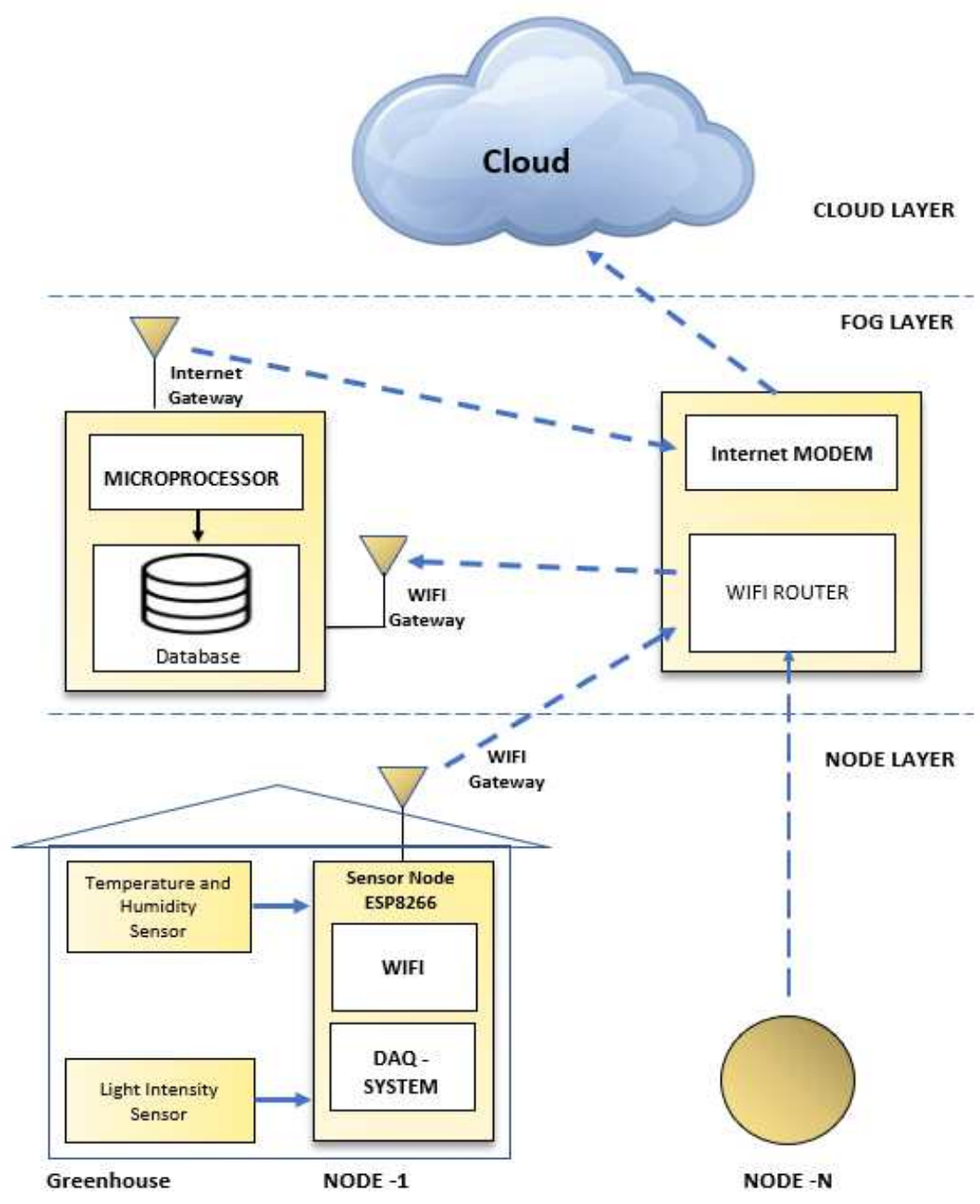

Fig. 2. The fog network system that has been revised for measurement physical parameters of plants in greenhouses.

In this study, fog server was built with a computer that was integrated with the database. On a computer fog server, a data sensor node service is created with the Message Queuing Telemetry Transport (MQTT) protocol 
to connect the two communication machines. Every data entered in the MQTT service is stored in the database, processed for specific purposes and sent to the internet using web-based programming. In this study the system performance was tested in terms of the precision of the sensor that was built, the speed of sending data and comparing the busyness of sending data in the cloud with the fog server and without using for the server.

\section{Result and Discussion}

The results of the design and realization of the fog networking system for monitoring the parameters of the physical chemistry of plants in the greenhouse were evaluated and analyzed through several stages of aiming. These tests include calibration of the measurement system from the sensor that has been read by the fog server. The test is carried out on the three parameters of the sensor that is used by comparing the measurement parameters with standard equipment on the greenhouse object and the results that have been read on the fog server. The two test results are then made a calibration graph as shown in Figure 3.

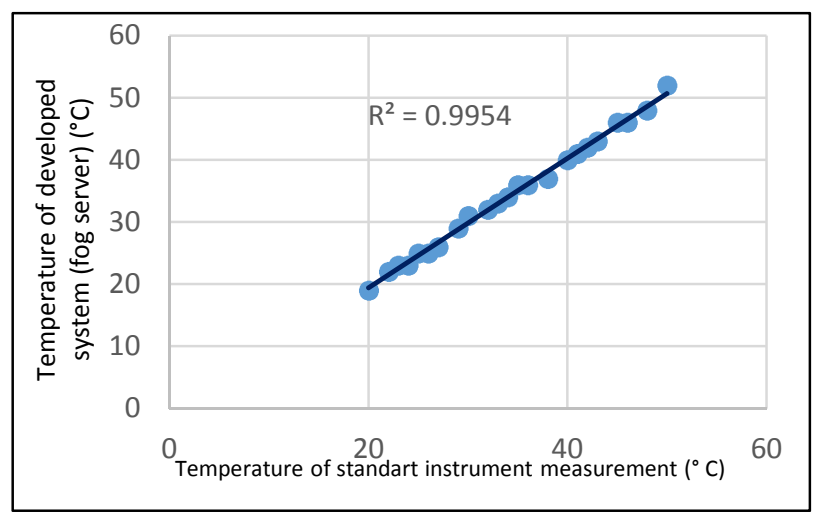

(a)

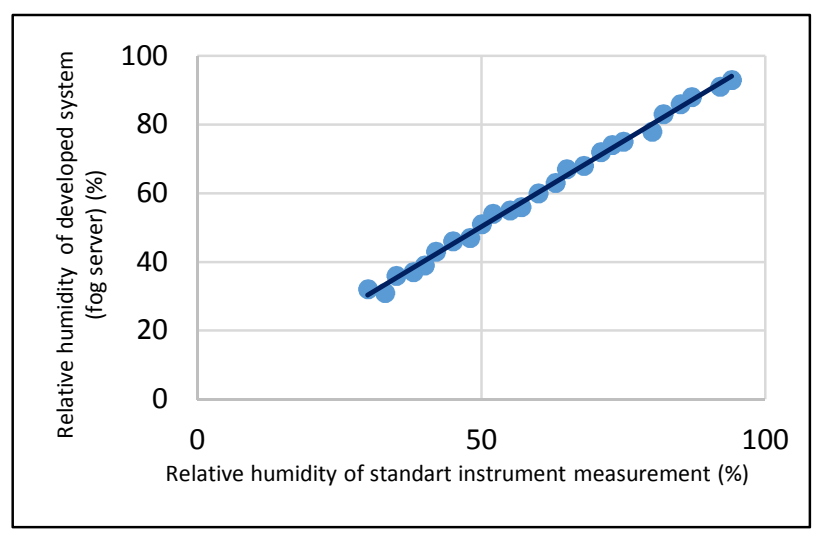

(b)

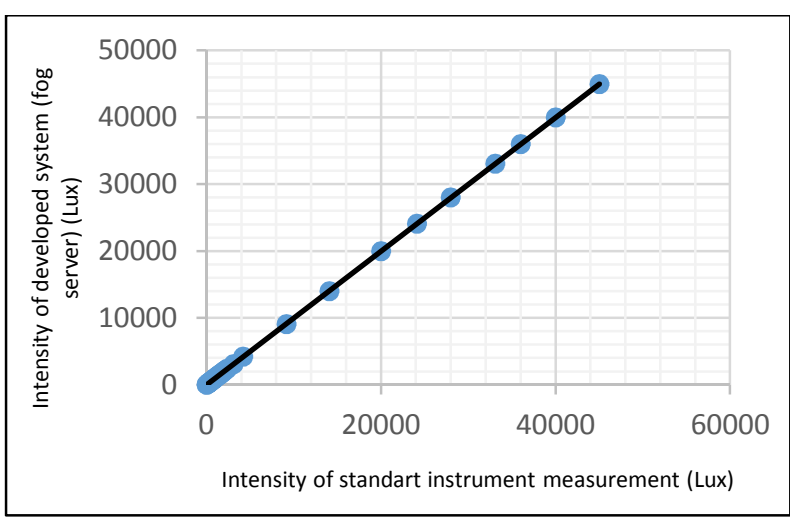

(c)

Fig. 3. The results of testing the measurement system parameters (a) Temperature, (b) Relative humidity and (c) Intensity of the greenhouse.

MAPE (Mean Absolute Percent Error) has been used to evaluate the accuracy of the measurement system with the formula:

$$
\mathrm{MAPE}=\frac{1}{N} \sum \frac{\mid \text { Actual }- \text { measurement } \mid}{\text { Actual }} \times 100 \%
$$

From the results of verification of the sensor measurements it can be seen that the average standard deviation value of each parameter is very small. This value reflects the difference in value between the standard measuring instrument used for calibration with a very small measurement system. As low as the MAPE values reflect the better the system is made [18].

In this study, fog network performance testing was carried out by testing the data input speed for 5 nodes. Each performance is compared to measurements for monitoring greenhouse parameters between using the Fog network and using cloud networks. The test results are shown in Figure 4. In the figure, the speed test value of the fog network compared to 1 node to 5 sensor nodes is compared.

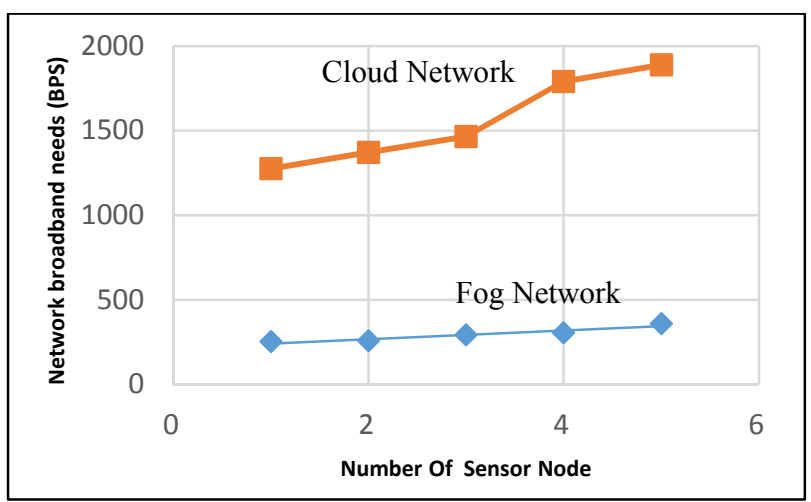

Fig. 4. Network broadband needs of sensor node on fog network and cloud network.

From the test results, it is obtained the results that for 1 node has an network broadband needs of 253 BPS for continuous data transmission. This speed is very low compared to the use of local broadband WIFI which is 
provided by 52 MBPS. In Figure 4 it is also explained that the speed of the fog network is relatively down but not significant if the sensor node is added. The addition of nodes causes the busyness of the communication protocol to become busier because it has to service data from various IP addresses. Network testing results have also been compared with cloud networks where sensor node data is sent directly using the internet network. Using cloud networks requires an network broadband need of 1276 BPS. The use of internet networks requires a higher broadband than the WIFI network as shown in Figure 4. This is due to the busyness and distance of the network to the physical object (sensor node) even further. In this study also tested the speed of sending data between fog networks and cloud networks. The test results are shown in the graph Figure 5.

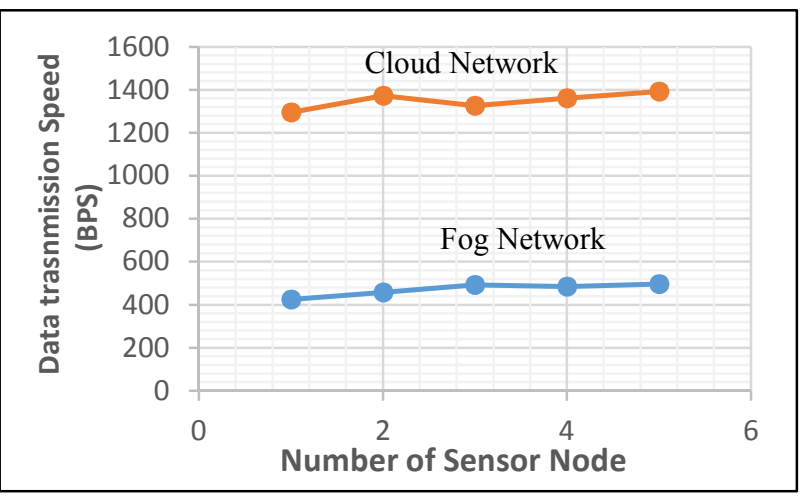

Fig. 4. Data transmission speed of sensor node on fog network and cloud network.

In general, the use of fog networks and cloud networks is not affected by the number of nodes. The transmission time test results show that the use of the fog network is much faster when compared to cloud networks. On fog networks the average data transmission speed is $471 \mathrm{~ms}$ while on cloud networks it is up to $1349 \mathrm{~ms}$. The speed is influenced by the network architecture where the use of fog networks, object measurement data from sensor is at a shorter network distance [19].

\section{Conclusion}

The fog network has been successfully built to monitor the physical parameters of plants in greenhouses, especially temperature, relative humidity and light intensity. The measurement system that has been built using the fog network has high accuracy with MAPE, namely temperature $=1.3 \%$, humidity: $1.9 \%$ and light intensity: $0.6 \%$. The use of the fog network has proven to not contribute significantly to the error value of measurement data sent to the server. The use of WIFI on the fog network requires less broadband when compared to cloud networks. The difference is very significant, which is an average of 253 BPS if using a fog network and 1276 BPS if using cloud networks. From experiments conducted, network usage is proven to have a high data transmission speed with $471 \mathrm{~ms}$ compared to the internet network with $1349 \mathrm{~ms}$. Variations in the number of sensor nodes up to 5 nodes do not significantly affect that speed.

\section{References}

1. C.R. Canavan, L. Graybill, W. Fawzi, J. Kinabo, Food Nutr. Bull 37, 1 (2017)

2. W. Nkomoki, M. Bavorová, J. Banout, J. Land Use Policy 78, November ( 2018)

3. J.A. Delgado, K. Kowalski, C. Tebbe, J. Computers and Electronics in Agriculture 91, February (2013)

4. J, Velicka, M, Pies, R. Hajovsky, IFAC-Papers On Line 51, 6(2018)

5. M. A. Campbell, Z. Li, J.W. Buck 100, October (2017)

6. M. Azaza, C. Tanougast, E. Fabrizio, A. Mami, ISA Transactions 61, March (2016)

7. D. Prehaten, S. Indrioko, S.N. Hardiwinoto, N. Na'iem, M. Supriyo, Journal of Forest Science 12, (2018)

8. R. Syam, W.A. Piarah, B. Jaelani, Internasional Journal on Smart Material and Mechatronics 2, (2015)

9. Syam, R., Piarah W.A. dan Jaelani B. Controlling Smart Green House Using Fuzzy Logic Method. Internasional Journal on Smart Material and Mmechatronics 2, (2015)

10. T. Wang, J. Zeng, Y. Lai, Y. Cai, H. Tian, Y. Chen, B. Wang, Future Generation Computers Systems, July (2017)

11. L. Peng, A.R Dhaini, P.H. Ho, 2017. J. Future Generation Computer Systems 88, November (2018)

12. D. Wu, S. Liu, L. Zhang, J. Terpenny, R.X. Gao T. Kurfess, J.A. Guzzo, 2017, Journal of Manufacturing Systems 43, (2017)

13. D. Wu, D.W. Rosen, L. Wang, D. Schaefer, Comput. Aided, Des (2015)

14. G. Paola, V. Naranjo, Z. Pooranian, M. Shojafar, M. Conti, R. Buyya, J. Parallel Distrib. Comput., July (2018)

15. A. Alrawais, A. Alhothaily, B. Mei, T. Song, X. Cheng, Procedia Computer Science 129, (2018)

16. X. S. XuLi, W. Tang, W. Zhang, Information Fusion 31, September (2016)

17. C. Lin, H. Chin, W. Chen, Journal of Network and Computer Applications 116, (2018)

18. S. Kim, H. Kim, International Journal of Forecasting 32, 3(2016)

19. D. Wu, S. Liu, L. Zhang, J. Terpenny, R. X. Gao, T. Kurfess, J. A. Guzzo, Journal of Manufacturing Systems 43, (2017) 